\title{
Diastereoselective Ring-Closing Metathesis to Build a Quaternary Carbon Center
}

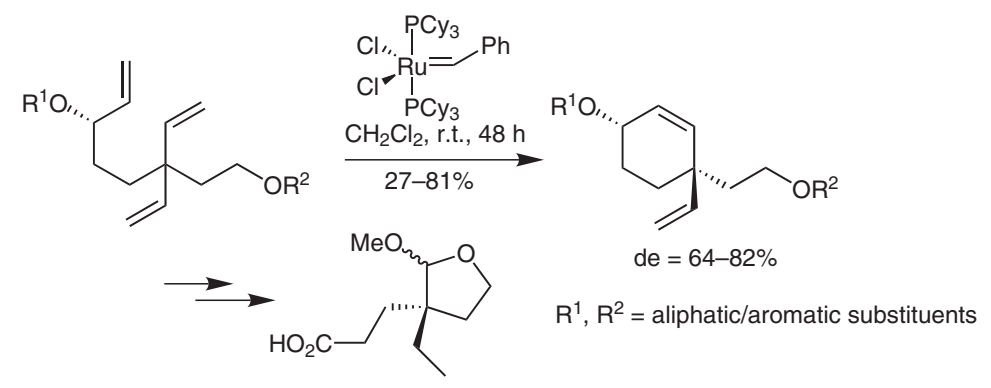

Significance: Diastereoselective ring-closing metathesis (RCM) was used to create a quaternary stereogenic center induced by protected hydroxyl groups. The initial stereogenic center was conveniently installed by Sharpless asymmetric epoxidation. The chiral RCM product cyclohexene was converted into a key chiral precursor used for the total synthesis of indole-containing alkaloid (-)-eburnamonine.
Comments: Stereoselective quaternary carbon center formation is still a problematic step in many natural product syntheses. The authors explored a RCM strategy to give a cyclohexene system with functionalizable groups on the quaternary center. Fine-tuning the selectivity by varying the protecting group on the hydroxy moieties gave a de of $81 \%$ using Grubbs' $1^{\text {st }}$-generation catalyst when $\mathrm{R}^{1}=\mathrm{TMS}, \mathrm{R}^{2}=\mathrm{Bz}$. Unfortunately, the conversion seems to be low for the substrates which provide the highest de's. 